\title{
Alterações fisiológicas de matrizes suínas criadas nas condições climáticas do semiárido mineiro
}

\author{
Physiological alteration of swine matrices created in the climatic semiarid conditions of Minas Gerais
}

\section{Thaís Emanuele Soares*, Cinara da Cunha Siqueira Carvalho, Camila Maida de Albuquerque Maranhão, Geruza Cardoso da Silva, Thamara Amaral Diniz, Kátia Cristiane Borges Pereira e Maria Cecilia Magalhães Gonçalves}

Recebido em 02/03/2016 / Aceito em 17/03/2017

\section{RESUMO}

A criação de suínos na região do semiárido mineiro possui caráter inicial, porém há perspectivas de crescimento, entretanto devido às altas temperaturas verificadas ao longo do ano a produção é reduzida. Diante disso, o objetivo deste trabalho foi avaliar o efeito do ambiente climático sobre os parâmetros fisiológicos de matrizes suínas antes e após o parto. O experimento foi conduzido em uma Granja representativa na criação de suínos localizada na região do Projeto Gorutuba, município de Nova Porteirinha, MG. Foram utilizadas seis matrizes alojadas em baias individuais. As variáveis climáticas temperatura de ar, umidade relativa do ar, temperatura de ponto de orvalho e temperatura de globo negro foram monitoradas por meio do uso de dois dataloggers. Os parâmetros fisiológicos avaliados foram: temperatura de superfície corporal, frequência respiratória, frequência cardíaca e temperatura retal. As variáveis ambientais e fisiológicas foram coletadas às 8:00, 11:00, 13:00, 15:00 e 17:00 h. Os dados foram submetidos à análise de variância, e quando significativos foram submetidos ao teste de Tukey a $5 \%$ de probabilidade para comparação entre as médias. De posse dos dados coletados verificou-se que entre às 13:00 h e 17:00 h e durante o período após o parto os animais ficaram expostos ao ambiente de desconforto térmico sendo este o fator responsável pela alteração na frequência respiratória, principalmente quando associado à ingestão de alimentos. Os valores médios $(\mathrm{p}<0,05)$ de temperatura de superfície corporal e retal foram superiores no período após o parto, porém não expressaram desconforto térmico das matrizes suínas. A criação desses animais na região do semiárido mineiro é possível, porém é necessário que sejam adotadas medidas como o uso de ventiladores e manejo de cortinas para minimizar o estresse calórico dos animais.

PALAVRAS-CHAVE: ambiência, conforto térmico, suinocultura.

\section{ABSTRACT}

The swine breeding in the Minas Gerais semiarid has an initial characterization, but there are prospects for growth, however due to the high temperatures verified throughout the year, production is reduced. Therefore, the objective of this work was to evaluate the effect of the climatic environment on the physiological parameters of swine matrices before and after farrowing. The experiment was carried out in a representative swine breeding farm located in the region of the Gorutuba Project, in Nova Porteirinha city, MG. Six swine matrices housed in individual bays were used. The climatic variables, air temperature, relative air humidity, dew point temperature and black globe temperature were monitored through the use of two dataloggers. The physiological parameters evaluated were: body surface temperature, respiratory rate, heart rate and rectal temperature. The environmental and physiological variables were collected at $8 \mathrm{am}, 11 \mathrm{am}, 01 \mathrm{pm}, 03 \mathrm{pm}$ and $05 \mathrm{pm}$. Data was submitted to analysis of variance, and when significant were submitted to the Tukey test at 5\% probability for comparison between the means. With the data collected, it was observed that between $01 \mathrm{pm}$ and $05 \mathrm{pm} \mathrm{h}$ and during the post-partum period, the animals were exposed to the thermal discomfort of the environment, being the responsible factor for the alteration in the respiratory rate, especially when associated with the ingestion of foods. The mean values $(p<0.05)$ of body and rectal surface

Universidade Estadual de Montes Claros, Montes Claros, MG, Brasil.

*Autor para correspondência <thathamanu@hotmail.com> 
temperature were higher in the postpartum period, but did not express thermal discomfort of the swine matrices. The raising of these animals in the semiarid Minas Gerais is possible; however, it is necessary to adopt measures such as the use of ventilators and the use of curtains to minimize the animal's caloric stress.

KEYWORDS: environment, thermal comfort, swine farming.

\section{INTRODUÇÃO}

A suinocultura no Brasil é uma atividade que se encontra em intenso desenvolvimento, e o desempenho zootécnico dos suínos apresentou melhoras nas últimas décadas, principalmente no número de suínos abatidos/porca/ano, possibilitando à indústria suinícola notável potencial para fornecer ao mercado consumidor fontes proteicas saudáveis a um baixo custo (COSTA JUNIOR et al. 2015). Entretanto, diversos fatores extrínsecos, como o clima, influenciam de forma negativa no sistema produtivo, devido à dificuldade que os suínos possuem em dissipar calor.

$\mathrm{O}$ estresse térmico durante a gestação e lactação, pode comprometer a ingestão de alimentos, levando à perda de peso com mobilização de gordura corporal, reduzindo a quantidade de leite produzido, aumentando a taxa respiratória e a temperatura retal e comprometendo o desenvolvimento da leitegada (WILLIAMS et al. 2013).

Sem um ambiente adequado, o animal é incapaz de demonstrar seu máximo potencial genético, de manter a salubridade e de nutrir-se de forma adequada, tanto em função do consumo, como também em função do aproveitamento de nutrientes, devido ao desvio de energia para a manutenção da temperatura corporal. Neste sentido, a manutenção do conforto térmico ambiental é de fundamental importância para se garantir o maior desempenho dos suínos, evitando assim que eles diminuam a eficiência de utilização da energia disponível (KERR et al. 2003, SARUBBI et al. 2008). Diante isso, o objetivo deste trabalho foi avaliar o efeito do ambiente climático sobre os parâmetros fisiológicos de matrizes suínas antes e após ao parto.

\section{MATERIAL E MÉTODOS}

O experimento foi conduzido em uma Granja representativa na criação de suínos localizada na região do Projeto Gorutuba, município de Nova Porteirinha, MG, região do semiárido mineiro. A área experimental está localizada a oito $\mathrm{km}$ da cidade de Janaúba, com latitude de $15^{\circ} 47^{\prime} 50^{\prime}$ 'S e longitude $43^{\circ} 18^{\prime} 31$ 'W, altitude de $516 \mathrm{~m}$ e clima, segundo Köppen, do tipo AW (tropical chuvoso, savana com inverno seco).

O experimento foi conduzido utilizando-se o delineamento inteiramente casualizado (DIC) com esquema fatorial $2 \times 5$, sendo 2 períodos (antes e após o parto) e 5 horários de avaliação (às 8:00, 11:00, 13:00, 15:00 e 17:00 h) em seis matrizes suínas durante 17 dias (repetições) consecutivos para cada animal. A realização da coleta dos dados experimentais ocorreu durante o período de outono, compreendendo o final da gestação e a fase de lactação das matrizes.

Os animais foram alojados em gaiolas individuais de $2,19 \mathrm{~m}$ de comprimento, por $0,55 \mathrm{~m}$ de largura, situadas em um galpão de alvenaria com cobertura de telha de barro, com piso de concreto e pé-direito de $3 \mathrm{~m}$, o galpão estava orientado na direção norte-sul. A sala da maternidade continha dois ventiladores localizados nas extremidades das paredes, sendo os mesmos acionados na parte da tarde, sem nebulizadores. As baias foram equipadas com comedouro e bebedouro tipo concha, não automático, e escamoteador para os leitões.

O manejo nutricional seguia o procedimento padrão da granja, na qual foi fornecido $1,5 \mathrm{~kg}$ de ração a base de milho e soja no período da manhã, e a mesma quantidade no período da tarde durante o período que antecedia o parto. Durante a lactação foi fornecido $3 \mathrm{~kg}$ de ração à base de milho e soja no período da manhã, e $3 \mathrm{~kg}$ na parte da tarde, sendo oferecida a ração úmida para esses animais, duas vezes por dia.

Foram monitoradas as variáveis meteorológicas na sala de maternidade, por meio do uso de dois data loggers Extech, modelo RHT-10, instalados na altura dos animais, permitindo a caracterização das seguintes variáveis climáticas: temperatura do ar (Tar), umidade relativa do ar (UR), temperatura de ponto de orvalho (Tpo) e temperatura de globo negro (Tgn).

De posse dos dados das variáveis climáticas, calculou-se o Índice de Temperatura de Globo e Umidade (ITGU) por meio da seguinte equação 1, proposta por BUFFINGTON et al. (1981):

$$
\mathrm{ITGU}=\mathrm{Tgn}+0,36 \mathrm{Tpo}+41,5 \quad \text { Equação } 1
$$


Em que:

ITGU = Índice de Temperatura de Globo Negro e Umidade;

Tgn $=$ Temperatura de globo negro, ${ }^{\circ} \mathrm{C}$;

Tpo $=$ Temperatura do ponto de orvalho, ${ }^{\circ} \mathrm{C}$.

Os parâmetros fisiológicos foram caracterizados por meio da temperatura de superfície corporal (TSC), frequência respiratória $(\mathrm{FR})$, frequência cardíaca $(\mathrm{FC})$ e temperatura retal (TR). A coleta de dados ocorreu às 8:00, 11:00, 13:00, 15:00 e 17:00 h. A temperatura retal foi obtida com o auxílio de um termômetro digital, DigiSense, modelo 8522-10.

A temperatura da superfície corporal foi obtida por meio das médias verificadas no tronco dos animais, por meio da utilização de um termômetro infravermelho.

A frequência respiratória foi obtida a partir da observação visual direta e quantificação dos movimentos do flanco por 15 segundos e posteriormente multiplicado o resultado por quatro para determinar a frequência respiratória por minuto.

A frequência cardíaca foi auscultada por meio do uso de um estetoscópio, colocado na parte ventral do animal, tendendo ao lado esquerdo do animal, próximo ao coração e mensurado durante 15 segundos para posterior multiplicação por quatro para determinar os batimentos cardíacos por minuto.

Para a análise estatística das variáveis climáticas e fisiológicas adotou-se o delineamento experimental inteiramente casualizado, sendo os dias utilizados como repetições. Para caracterizar o desempenho das variáveis climáticas foi utilizado o teste $\mathrm{F}$ para comparar o efeito dos horários de avaliação em cada tratamento, utilizando a análise de regressão (contrastes ortogonais). As variáveis fisiológicas foram submetidas à análise de variância por meio do teste $\mathrm{F}$ e quando significativo, as médias dos tratamentos foram comparadas pelo teste de Tukey $(\mathrm{p}<0,05)$ utilizando o programa estatístico SISVAR $^{\circledR}$ (FERREIRA 2008).

\section{RESULTADOS E DISCUSSÃO}

De acordo com CAMPOS et al. (2009) matrizes suínas na fase de gestação e lactação devem ser criadas em ambiente onde a temperatura do ar esteja na faixa de 12 a $18{ }^{\circ} \mathrm{C}$ e a umidade relativa de 50 a $70 \%$. Na Tabela 1 verifica-se que os valores médios $(\mathrm{p}<0,05)$ de temperatura do ar foram superiores aos valores sugeridos por CAMPOS et al. (2009), e no período de 13:00 h às 17:00 h, a Tar foi superior na fase de pós-parto.

A umidade relativa do ar foi decrescente nos dois períodos até às 13:00 h, e os menores valores médios $(p<0,05)$ foram verificados durante o pósparto. A umidade relativa esteve dentro dos valores considerados ideais por CAMPOS et al. (2009), com

Tabela 1. Temperatura do ar $\left({ }^{\circ} \mathrm{C}\right)$, umidade relativa do ar $(\%)$ e índice de temperatura de globo e umidade (ITGU) (valores médios) antes e após o parto.

Table 1. Air temperature $\left({ }^{\circ} \mathrm{C}\right)$, relative humidity $(\%)$ and globe temperature and humidity index (ITGU) (means values) before and after farrowing.

\begin{tabular}{|c|c|c|c|c|c|c|c|}
\hline \multicolumn{8}{|c|}{ Temperatura do ar } \\
\hline \multirow{2}{*}{ Período } & \multicolumn{5}{|c|}{ Horário } & \multirow[t]{2}{*}{ Equação } & \multirow[t]{2}{*}{$\mathrm{R}^{2}(\%)$} \\
\hline & 08:00 & $11: 00$ & $13: 00$ & $15: 00$ & $17: 00$ & & \\
\hline Antes do parto & $26,4 a$ & $30,3 \mathrm{a}$ & $32,3 \mathrm{a}$ & $31,0 \mathrm{a}$ & $29,8 \mathrm{a}$ & $\hat{\mathrm{y}}=-2,077506+4,934429 \mathrm{x}-$ & \multirow{2}{*}{99,30} \\
\hline Após o parto & $26,2 \mathrm{a}$ & $29,8 \mathrm{a}$ & $32,8 \mathrm{a}$ & $32,7 \mathrm{~b}$ & $30,8 b$ & $0,176231 x^{2}$ & \\
\hline \multicolumn{8}{|c|}{ Umidade Relativa } \\
\hline Antes do parto & $70,3 \mathrm{a}$ & $59,7 \mathrm{~b}$ & $51,5 b$ & $55,8 \mathrm{~b}$ & $57,7 \mathrm{~b}$ & $\hat{y}=164,461479-16,417248 x+$ & \multirow{2}{*}{97,47} \\
\hline Após o parto & $69,9 \mathrm{a}$ & $54,2 \mathrm{a}$ & $47,5 \mathrm{a}$ & $47,5 \mathrm{a}$ & $52,5 \mathrm{a}$ & $0,581204 x^{2}$ & \\
\hline \multicolumn{8}{|l|}{ ITGU } \\
\hline Antes do parto & $74,8 \mathrm{a}$ & $78,5 \mathrm{a}$ & $81,0 \mathrm{a}$ & $80,5 \mathrm{a}$ & $78,8 \mathrm{a}$ & $\hat{y}=45,996713+5,099272 x-$ & \multirow{2}{*}{96,12} \\
\hline Após o parto & $75,4 \mathrm{a}$ & $79,2 \mathrm{a}$ & $81,9 b$ & $81,8 b$ & $79,6 \mathrm{a}$ & $0,183071 x^{2}$ & \\
\hline
\end{tabular}

Médias seguidas de letras distintas na mesma coluna, diferem entre si $(\mathrm{p} \leq 0,05)$ pelo teste Tukey. 
pequenas variações $(47,5 \%)$ que não impedem a dissipação de calor corporal.

A junção da temperatura do ar com a umidade relativa informa a faixa de conforto térmico ambiental a qual os animais estão expostos (Figura 1). De acordo com BUFFINGTON et al. (1981), valores de ITGU de até 74 definem condição de conforto no ambiente, de 74 a 78 é sinal de alerta, de 79 a 84 é sinal de perigo e, acima de 84 é considerado sinal de emergência. Os valores médios de ITGU obtidos caracterizaram o ambiente climático em situação de perigo a partir das $11: 00 \mathrm{~h}$ e os maiores valores médios $(\mathrm{p}<0,05)$ foram verificados entre às 13:00 e 15:00 h no período após o parto.

Com relação às respostas fisiológicas dos animais avaliados, verificou-se que a temperatura de superfície corporal foi superior no período de pós-parto, contudo os valores médios das duas fases $(p<0,05)$ estiveram de acordo com os verificados por NÄÄS et al. (2013), que ao avaliarem suínos em ambiente com ventilação natural e resfriamento adiábatico a TSC variou de $31,68{ }^{\circ} \mathrm{C}$ à $36,51{ }^{\circ} \mathrm{C}$. Neste trabalho a TSC das matrizes variou de $32{ }^{\circ} \mathrm{C}$ a $35^{\circ} \mathrm{C}$. No período após o parto, quando o ITGU máximo foi de 81,9 (condição de perigo) a TSC foi inferior à verificada por NÄÄS et al. (2013).

Não houve diferença significativa $(p<0,05)$ para a frequência respiratória antes e após o parto nos horários entre 08:00 e 15:00 h. Houve diferença $(\mathrm{p}<0,05)$ as 17:00 $\mathrm{h}$ em virtude do aumento do calor metabólico devido à ingestão de alimento, assim como às 08:00 $\mathrm{h}$ como pode-se observar por meio da equação cúbica de regressão. MARTINS et al. (2008) avaliando a FR de matrizes suínas na fase de lactação verificaram a variação de 61,34 mov $\min ^{-1}$ a 82,33 mov $\min ^{-1}$, e com base na matriz de correlação a FR foi mais vulnerável às variações ambientais, especialmente à temperatura do ar, sendo este mecanismo, o mais importante para dissipação de calor e manutenção da homeotermia, em matrizes suínas lactantes. Por meio da análise de regressão (Tabela 2) verifica-se que a partir das 13:00 h a FR assumiu valores superiores à $82 \mathrm{mov} \mathrm{min}^{-1}$, sendo que às 15:00 h quando o ITGU era de 81,8 , a FR foi de até 94,6 $\mathrm{mov}^{\mathrm{min}^{-1}}$ demonstrando assim o efeito do ambiente climático sobre o aumento da frequência respiratória das matrizes suínas.

Não houve diferença significativa $(p<0,05)$ para a frequência cardíaca antes e após o parto. A FC se ajusta para atender a demanda de cada situação

ITGU antes $\square$ ITGU depois — Tar antes — Tar depois —UR antes —UR depois

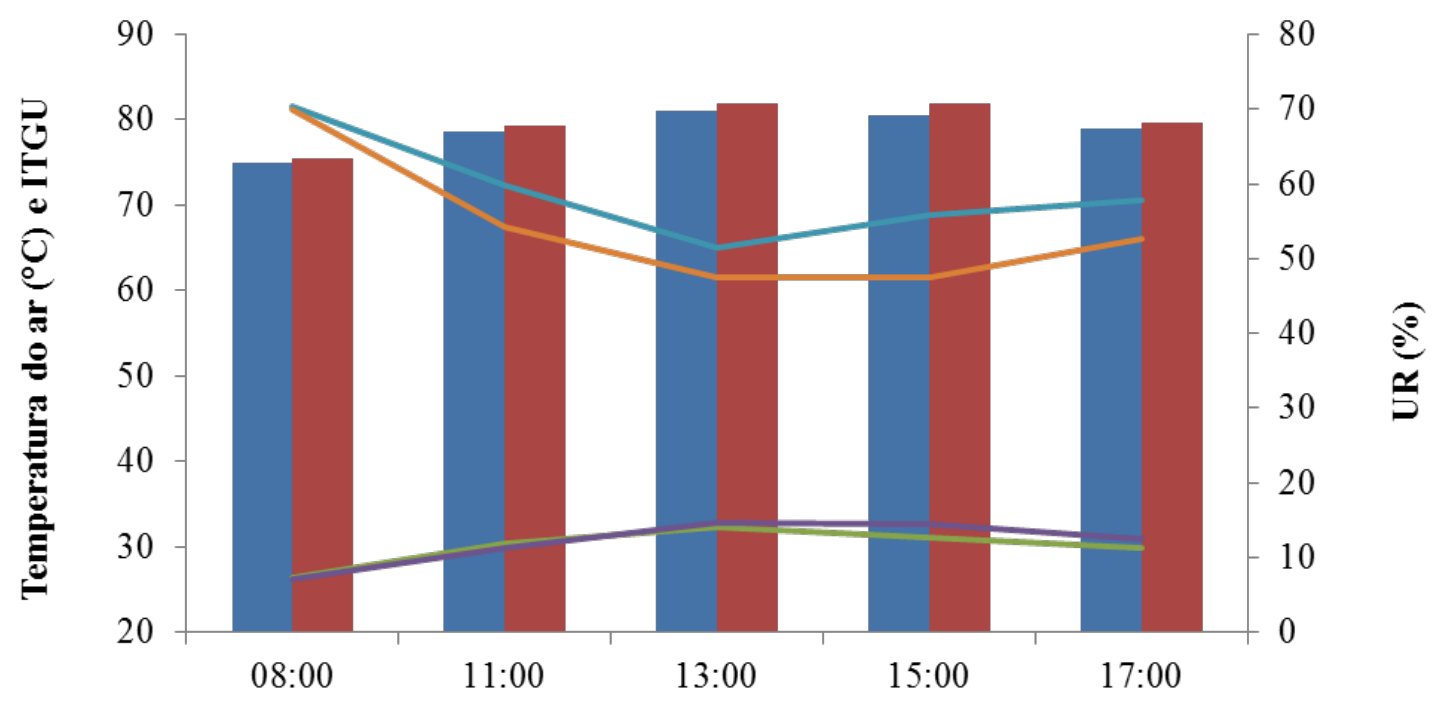

Horários de coleta

Figura 1. Análise conjunta da temperatura do ar (Tar), umidade relativa (UR) do ar e índice de temperatura de globo e umidade (ITGU) para os períodos, antes e após o parto das matrizes.

Figure 1. Joint analysis of air temperature (Tar), relative humidity (UR) and globe temperature and humidity index (ITGU) for the periods before and after farrowing. 
Tabela 2. Temperatura de superfície corporal $\left({ }^{\circ} \mathrm{C}\right)$, frequência respiratória $\left(\mathrm{mov} \min ^{-1}\right)$, frequência cardíaca (bat $\left.\min ^{-1}\right)$ e temperatura retal $\left({ }^{\circ} \mathrm{C}\right)$ (valores médios) de matrizes suínas antes e após o parto.

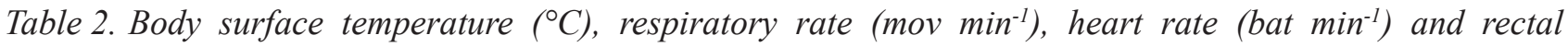
temperature $\left({ }^{\circ} \mathrm{C}\right)$ (mean values) of sows before and after farrowing.

\begin{tabular}{|c|c|c|c|c|c|c|c|}
\hline \multicolumn{8}{|c|}{ Temperatura Corporal } \\
\hline \multirow{2}{*}{ Período } & \multicolumn{5}{|c|}{ Horário } & \multirow[t]{2}{*}{ Equação } & \multirow[t]{2}{*}{$\mathrm{R}^{2}(\%)$} \\
\hline & 08:00 & $11: 00$ & $13: 00$ & $15: 00$ & $17: 00$ & & \\
\hline Antes do parto & $32.0 \mathrm{a}$ & $32.9 \mathrm{a}$ & $34.4 \mathrm{a}$ & $34.2 \mathrm{a}$ & $33.3 \mathrm{a}$ & $\hat{y}=26.659201+1.028810 X-$ & \multirow{2}{*}{89.49} \\
\hline Após o parto & $33.0 \mathrm{~b}$ & $33.7 \mathrm{~b}$ & $35.0 \mathrm{~b}$ & $34.9 \mathrm{~b}$ & $35.0 \mathrm{~b}$ & $0.032402 \mathrm{X}^{2}$ & \\
\hline \multicolumn{8}{|c|}{ Frequência Respiratória } \\
\hline Antes do parto & $71.7 \mathrm{a}$ & $65.7 \mathrm{a}$ & $82.1 \mathrm{a}$ & $94.6 \mathrm{a}$ & $71.7 \mathrm{a}$ & $\hat{y}=582.733402-136.197244 X+$ & \multirow{2}{*}{97.50} \\
\hline Após o parto & $72.8 \mathrm{a}$ & $67.8 \mathrm{a}$ & $87.3 \mathrm{a}$ & $93.1 \mathrm{a}$ & $93.6 b$ & $11.495911 \mathrm{X}^{2}-0.305514 \mathrm{X}^{3}$ & \\
\hline \multicolumn{8}{|c|}{ Batimentos Cardíacos } \\
\hline Antes do parto & $89.4 a$ & $91.7 \mathrm{a}$ & $87.2 \mathrm{a}$ & $92.0 \mathrm{a}$ & $101.0 \mathrm{a}$ & $\hat{\mathrm{y}}=142.319595-14.349893 \mathrm{X}+$ & \multirow{2}{*}{99.73} \\
\hline Após o parto & $89.4 \mathrm{a}$ & $90.0 \mathrm{a}$ & $93.9 \mathrm{a}$ & $96.6 \mathrm{a}$ & $95.5 \mathrm{a}$ & $1.217510 \mathrm{X}^{2}-0.031240 \mathrm{X}^{3}$ & \\
\hline \multicolumn{8}{|c|}{ Temperatura Retal } \\
\hline Antes do parto & $37.0 \mathrm{a}$ & $37.2 \mathrm{a}$ & $37.8 \mathrm{a}$ & $37.9 \mathrm{a}$ & $37.7 \mathrm{a}$ & $\hat{y}=35.562033+0.404709 X-$ & \multirow{2}{*}{77.67} \\
\hline Após o parto & $38.3 \mathrm{~b}$ & $38.4 \mathrm{~b}$ & $39.1 \mathrm{~b}$ & $39.1 \mathrm{~b}$ & $38.9 \mathrm{~b}$ & $0.012489 X^{2}$ & \\
\hline
\end{tabular}

Médias seguidas de letras distintas na mesma coluna, diferem entre si $(\mathrm{p} \leq 0,05)$ pelo teste Tukey.

fisiológica, e em condições de estresse pode chegar a 196 bat $\mathrm{min}^{-1}$ enquanto que em repouso, as matrizes suínas apresentam 91 bat min $^{-1}$ (DALLA COSTA et al. 2008, BAPTISTA et al. 2011). Neste trabalho a FC variou de 89,4 e 101 bat $\min ^{-1}$ (Tabela 2), esse fato esclarece que o ambiente climático e o processo de pré e pós-parto não alteraram a $\mathrm{FC}$ dos animais avaliados.

Com relação à temperatura retal (Tabela 2), observou-se que os valores aferidos tanto para o período que antecede como após o parto, estiveram dentro dos padrões recomendados por MUIRHEAD \& ALEXANDER (1997), nos quais matrizes na gestação podem ter a temperatura retal por volta de $38,6{ }^{\circ} \mathrm{C} \pm 1,5^{\circ} \mathrm{C}$, enquanto que na fase de lactação a TR pode ter um aumento médio até os $39,1{ }^{\circ} \mathrm{C} \pm$ $1,5{ }^{\circ} \mathrm{C}$. Contudo, no período pós-parto a TR foi superior, uma vez que a maior atividade metabólica na fase de lactação, com aumento do consumo alimentar e síntese de leite, aumenta a produção de calor corporal, em comparação com o período anterior ao parto (MARTINS et al. 2008).

\section{CONCLUSÃO}

Matrizes suínas durante o período que antecede e após o parto alteram a frequência respiratória em função do ambiente climático e ingestão de alimentos. A temperatura de superfície corporal e retal são maiores durante o período após o parto, porém não promoveram desconforto térmico às matrizes suínas. Assim, a criação desses animais na região do semiárido mineiro é possível, porém é necessário que sejam adotadas medidas como o uso de ventiladores e manejo de cortinas para minimizar o estresse calórico dos animais.

\section{REFERÊNCIAS}

BAPTISTA RIAA et al. 2011. Indicadores do bem-estar em suínos. Ciência Rural 41: 1823-1830.

BUFFINGTON DE et al. 1981. Black globe humidity index (BGHI) as comfort equation for dairy cows. Transacofthe ASAE 24: 711-714.

CAMPOS JA et al. 2009. Qualidade do ar, ambiente térmico e desempenho de suínos criados em creches com dimensões diferentes. Engenharia Agrícola 29: 339-347. COSTA JUNIOR MB et al. 2015. Torta da polpa da 
macaúba para suínos em terminação. Revista Brasileira de Saúde e Produção Animal 16: 325-336.

DALLA COSTA OA et al. 2008. Tempo de jejum na granja sobre o perfil hormonal e os parâmetros fisiológicos em suínos de abate pesados. Ciência Rural 38: 2300-2306.

KERR BJ et al. 2003. Influences of dietary protein level, amino acid supplementation and environmental temperature on performance, body composition, organ weights and total heat production of growing pigs. Journal of Animal Sciences 81: 1998-2007.

MARTINS TDD et al. 2008. Respostas termorreguladoras de matrizes suínas híbridas em lactação, mantidas em ambiente quente. Ciência e Agrotecnologia 32: 961-968.

MUIRHEAD MR \& ALEXANDER TJL. 1997 Managing pig health and the treatement of disease. Sheffield: $5 \mathrm{M}$ Entreprises. 608p.

NÄÄS IA et al. 2013. Temperatura superficial de porcas em lactação submetidas ao resfriamento adiabático. Enciclopédia Biosfera 9: 2006-2013.

SARUBBI $J$ et al. 2008. Power-saving procedures and animal thermal comfort at a growing/finishing swine production unit. Revista Brasileira de Engenharia de Biossistemas 2: 185-192.

WILLIAMS AM et al. 2013. Effects of a controlled heat stress during late gestation, lactation, and after weaning on thermoregulation, metabolism, and reproduction of primiparous sows. Journal of Animal Sciences 91: 27002714. 